This is an author manuscript of a paper published in a final edited form as:

Leuk Lymphoma. 2012 Dec;53(12):2474-8. [doi: 10.3109/10428194.2012.696313]

Chronic myeloid leukemia stem cells display alterations in expression of genes involved in oxidative phosphorylation.

Flis K, Irvine D, Copland M, Bhatia R, Skorski T.

available at: http://informahealthcare.com/doi/abs/10.3109/10428194.2012.696313 


\section{Chronic myeloid leukemia stem cells display alterations in the expression of genes involved in oxidative phosphorylation}

Running title: Altered oxidative phosphorylation in CML

Krzysztof Flis ${ }^{1,2}$, David Irvine ${ }^{3}$, Mhairi Copland ${ }^{3}$, Ravi Bhatia ${ }^{4}$, Tomasz Skorski ${ }^{1, *}$

${ }^{1}$ Department of Microbiology and Immunology, Temple University, School of Medicine, Philadelphia, PA 19140, USA

${ }^{2}$ Institute of Biochemistry and Biophysics, Polish Academy of Sciences, Warsaw, Poland

${ }^{3}$ Paul O'Gorman Leukemia Research Centre, University of Glasgow, Glasgow, UK.

${ }^{4}$ Division of Hematopoietic Stem Cell and Leukemia Research, Department of Hematology and Hematopoietic Cell Transplantation, City of Hope National Medical Center, Duarte, CA 91010, USA

${ }^{*}$ Corresponding author: Tomasz Skorski, Department of Microbiology and Immunology, Temple University School of Medicine, 3400 N. Broad Street, MRB 548, Philadelphia, PA 19140, USA, e-mail: tskorski@temple.edu

Key words: CML leukemia stem cells, oxidative phosphorylation, microarray analysis 


\begin{abstract}
Mitochondrial respiratory chain (MRC) consists of the protein complexes I, II, III, IV, and V to carry oxidative phosphorylation (OXPHOS), which depends on electron transport to generate ATP. Electron "leakage" from MRC generates reactive oxygen species (ROS). Chronic myeloid leukemia in chronic phase (CML-CP) stem cells (LSCs) produce high levels of mitochondrial ROS causing oxidative DNA damage resulting in genomic instability generating imatinibresistant BCR-ABL1 kinase mutants and additional chromosomal aberrations. Using global mRNA microarray analysis combined with Ingenuity pathway analysis we found that LSCs display enhanced expression of genes encoding MRC complexes I, II, IV, and V. However, expression of genes encoding complex III was deregulated. Treatment with imatinib did not correct the aberrant levels of MRC genes. Therefore we postulate that abnormal expression of MRC genes may facilitate electron "leakage" to promote production of ROS and accumulation of genomic instability in LSCs in imatinib-naive and imatinib-treated patients.
\end{abstract}




\section{Introduction}

Chronic myeloid leukemia in chronic phase (CML-CP) is initiated by the oncogenic BCR-ABL1 tyrosine kinase that transforms hematopoietic stem cells (HSCs) to leukemia stem cells (LSCs) [1]. Deregulated growth of LSCs-derived leukemia progenitor cells (LPCs) leads to the manifestation of the disease. ABL1 tyrosine kinase inhibitors (TKIs), such as imatinib, dasatinib and nilotinib may induce favorable therapeutic responses, but LSCs are intrinsically insensitive to TKIs despite inhibition of BCR-ABL1 kinase [2]. CML-CP cells may at some stage acquire additional genetic changes that confer TKI resistance and induce more aggressive CML blast phase (CML-BP) [3].

Genomic instability may result from enhanced oxidative DNA damage caused by reactive oxygen species (ROS) [4]. Superoxide anion $\left(\cdot \mathrm{O}_{2}{ }^{-}\right)$, the first produced ROS molecule, is a moderately stable free radical. Superoxide dismutase uses $\cdot \mathrm{O}_{2}^{-}$to generate hydrogen peroxide $\left(\mathrm{H}_{2} \mathrm{O}_{2}\right)$, which may be then converted to the highly reactive hydroxyl group $(\cdot \mathrm{OH})$. ROS can damage DNA bases to produce oxo-derivatives such as 7,8-dihydro-8-oxo-2'deoxyguanosine (8-oxoG) and also induce spontaneous DNA double-strand breaks (DSBs). Unfaithful and/or inefficient repair of these oxidative DNA lesions can generate point mutations and chromosomal aberrations.

We reported recently that CML-CP LSCs and early LPCs accumulate high levels of ROS resulting in accumulation of TKI-resistant BCR-ABL1 kinase mutants and additional chromosomal aberrations [5]. This effect was due to Rac2-mediated modification of the mitochondrial membrane potential $\left(\Delta \Psi_{m}\right)$ associated with slower electron flow through mitochondrial respiratory chain (MRC) complex III. This phenomenon facilitated electron "leakage" from the complex III and production of superoxide anion. The current study suggests 
that an imbalance in the expression of MRC components may contribute to production of ROS in CML-CP LSCs. 


\section{Materials and Methods}

\section{Isolation of hematopoietic stem and progenitor cell populations from CML patients and}

normal donors: Fresh leukapheresis or peripheral blood samples were obtained with written informed consent and approval of the Greater Glasgow and Clyde Ethics Committee (Institutional Review Board) from patients with newly diagnosed chronic phase $(n=6)$, accelerated phase $(n=4)$ or myeloid blast crisis $(n=2) C M L$ and healthy donors of peripheral blood stem cells $(n=3)$. Samples were enriched for CD34 ${ }^{+}$cells using CliniMACS (Miltenyi Biotec Ltd) according to the manufacturer's instructions and cryopreserved in $10 \%(\mathrm{v} / \mathrm{v})$ DMSO in $5 \%(w / v)$ Human Albumin Solution (Baxter Healthcare Ltd). After thawing, CD34 ${ }^{+}$cells were cultured overnight in serum free medium (SFM) consisting of Iscove's Modified Dulbecco's Medium (IMDM, Sigma-Aldrich) containing a serum substitute (bovine serum albumin, insulin, transferrin; all from StemCell Technologies), 0.1 $\mu \mathrm{M}$ 2-mercaptoethanol (Sigma-Aldrich), and a high concentration five growth factor cocktail comprising 100ng/mL Flt3-ligand (FIt3L), $100 \mathrm{ng} / \mathrm{mL}$ stem cell factor (SCF), and $20 \mathrm{ng} / \mathrm{mL}$ each of interleukin (IL)-3, IL-6 (all from StemCell Technologies) and granulocyte-colony stimulating factor (G-CSF; Chugai Pharma Europe). $\mathrm{CD} 4^{+} \mathrm{CML}$ and normal cells were simultaneously stained with lineage (Lin) cocktail-FITC, CD90-PE, CD34-PerCP, CD38-PE-Cy7, CD123-APC and CD45RA-Pacific Blue (all from BD Biosciences) at room temperature for 20 minutes. Unbound antibodies were washed off with $\mathrm{PBS} / 2 \%$ fetal calf serum (FCS) and the cells were sorted to isolate the different subpopulations (hematopoietic stem cell [HSC] $-\operatorname{lin}^{-} \mathrm{CD} 34^{+} \mathrm{CD} 38^{-} \mathrm{CD} 90^{+}$; multipotent progenitor [MPP] $-\operatorname{lin}^{-}$ CD34 ${ }^{+} \mathrm{CD} 38^{-} \mathrm{CD} 90^{-}$; common myeloid progenitor [CMP] - lin ${ }^{-C D} 34^{+} \mathrm{CD} 8^{+} \mathrm{CD} 123^{+} \mathrm{CD} 45 \mathrm{RA}^{-}$; granulocyte monocyte progenitor [GMP] $-\operatorname{lin}^{-} \mathrm{CD} 34^{+} \mathrm{CD} 38^{+} \mathrm{CD} 123^{+} \mathrm{CD} 45 \mathrm{RA}{ }^{\mathrm{lo}}$; and megakaryocyte erythroid progenitor $\left.[\mathrm{MEP}]-\operatorname{lin}^{-} \mathrm{CD} 34^{+} \mathrm{CD} 38^{+} \mathrm{CD} 123^{-} \mathrm{CD} 45 \mathrm{RA}^{-}\right)$using FACSAria (BD Biosciences). 
RNA preparation from normal and CML samples and microarray analysis: RNA was prepared from sorted subpopulations of cells using the RNeasy kit (Qiagen). RNA processing and microarray analysis was carried out at the Sir Henry Wellcome Functional Genomics Facility (SHWFGF, University of Glasgow). RNA samples were analyzed on the Affymetrix GeneChip Human Gene 1.0 ST arrays using standard Affymetrix protocols. The raw data CEL files were normalized using gcRMA n Partek Genomic Suite software http://www.partek.com/partekgs. One way ANOVA was performed on the imported, normalized and summarized microarray data to find differently expressed genes between all cell subpopulations and disease states. Lists of differentially expressed genes were created using an FDR limit of 0.05 to control for multiple comparisons but no limit on degree of fold change. These lists were uploaded directly into Ingenuity Pathway Analysis (IPA) software (Ingenuity Systems). To determine which functional groupings of genes were differentially expressed we performed gene ontology ANOVA (GO ANOVA). Partek GS utilizes the gene ontology (GO) database to map genes to standardized functional groupings. The ANOVA model was configured to contrast the different sub-populations and disease states in order to determine the significance and average fold change of each GO category across all the comparator groups. These results were visualized using dot plots where the average expression of all genes in that functional group is represented as a single dot. The average expression is plotted on a log2 scale on the $y$ axis, and each subpopulation in each disease state on the $\mathrm{x}$ axis.

Analysis of CML-CP samples treated or not with imatinib: CML-CP patient samples, imatinib treatment and microarray analysis were as described before [6]. Briefly, CML $\mathrm{CD} 4^{+} \mathrm{CD} 38^{-}$cells selected with flow cytometry sorting were treated with $1 \mu \mathrm{M}$ imatinib or cultured without exposure to the drug (control) for $24 \mathrm{hr}(\mathrm{n}=3$ each). Total RNA from 5000 cells was extracted with the RNeasy kit (Qiagen), amplified and labeled with GeneChip Two-Cycle Target Labeling and Control Reagents (Affymetrix, Santa Clara, CA), and hybridized to 
Affymetrix GeneChip Human Genome U133 Plus 2.0 Arrays. Microarray data analyses were performed with $\mathrm{R}$ (version 2.9) with genomic analysis packages (version 2.4) from Bioconductor. Expression data were normalized with the robust multiarray average (RMA) algorithm, with background adjustment, quantile normalization, and median polish summarization. Probe sets with low expression levels or low variability across samples were filtered. For genes with multiple probe sets, the gene level expression was set to be the median of the probe sets. Linear regression was used to model the gene expression with the consideration of 232 factorial design and matched samples. Differentially expressed genes were identified by calculating empirical Bayes moderated t-statistic, and $p$ values were adjusted by FDR with the "LIMMA" package. Gene Set Enrichment Analysis (GSEA) was performed with GSEA software version 2.04 (http://www.broadinstitute.org/gsea/) for detection of enrichment of predetermined gene sets with t-scores from all 13,812 genes [7]. These lists were uploaded directly into IPA software (Ingenuity Systems). 


\section{Results}

We performed global gene expression studies on normal and CML hematopoietic stem and progenitor cell populations (HSC, MPP, CMP, GMP and MEP) using the Affymetrix GeneChip Human Gene 1.0 ST arrays. One way ANOVA and GO ANOVA were used to identify differentially expressed single genes and functional groupings of genes, respectively, in comparison to normalized data from HSCs obtained from healthy donors. The gene grouping and individual genes associated with OXPHOS (GO-ID: 6119) such as UQCRB, UQCRC1, UQCRH were significantly de-regulated in CML-CP LSCs compared to normal HSCs, but were not abundantly altered between normal and CML-CP in the different progenitor sub-populations (Figure 1A, B; Supplemental Table 1). As the disease progressed to CML-AP and CML-BP, expression of OXPHOS genes was highly variable and became more often downregulated in LSCs and LPCs.

Differential expression of genes involved in OXPHOS in CML-CP LSCs compared to normal HSCs was further analyzed using Ingenuity Functional Term (IFT): Oxidative phosphorylation (Figure 2; Supplemental Table 2) and Mitochondrial Dysfunction (Figure 3;

Supplemental Table 3). Both approaches detected uniform upregulation of the expression of genes encoding MRC complex I (NADH-coenzyme Q oxidoreductase) such as NDUFA2, NDUFA3, NDUFA9, NDUFA11, NDUFB6, NDUFB9, NDUFB10, NDUFB11, NDUFAB1, NDUFS4, NDUFS7, NDUFS8 and NDUFV3, MRC complex II (succinate-Q oxidoreductase) such as SDHB, MRC complex IV (cytochrome c oxidase) such as COX5A, COX5B, COX6B1, COX7B and COX11, and MRC complex V (ATP synthase) such as ATP5A1, ATP5B, ATP5C1, ATP5D, ATP5G1, ATP5H, ATP5I and ATP5J2. In contrast, expression of genes encoding MRC complex III (Q-cytochrome c oxidoreductase) were in disarray: CYTB and UQCRB were downregulated whereas CYC1, UQCR10, UQCRC1, UQCRC2, UQCRFS1 and UQCRH were upregulated. 
Ingenuity Pathway Analysis (IPA), including the genes identified by IFT, highlighted potential deregulation of the MRC genes in CML-CP LSCs (Figure 4A). Interestingly, imatinib treatment did not correct their aberrant expression (Figure 4B; Supplemental Table 4). 


\section{Discussion}

Some cancer stem cells (CSCs), for example breast and lung CSCs, maintain low ROS levels due to several factors, including increased expression of free radicals scavengers, lower quantity of mtDNA and higher $\Delta \Psi_{m}[8,9]$. In contrast, LSCs from BCR-ABL1 -positive CML-CP and IDH1/2 mutation -positive AML accumulate high levels of ROS $[5,10]$. We reported that high levels of ROS in CML-CP LSCs induced oxidative DNA damage resulting in genomic instability which may produce imatinib-resistant BCR-ABL1 kinase mutants and additional chromosomal aberrations leading to the disease relapse and/or malignant progression $[5,11,12]$. Since CML-CP LSCs, in particular the quiescent sub-population, are refractory to TKIs, genomic instability in these cells is especially dangerous [13]. Identification of the mechanisms of genomic instability in CML-CP LSCs may lead to novel therapeutic modalities preventing the disease relapse/malignant progression in patients under TKI treatment.

At least $50 \%$ of ROS in CML-CP LSCs is produced by mitochondria [5]. However, the mechanism responsible for this phenomenon has not been described. Here we report that CML$\mathrm{CP}$ display deregulated expression of the MRC components, which may contribute to overproduction of mitochondrial ROS. Numerous MRC genes appear overexpressed in LSCs suggestimg enhanced OXPHOS, which may reflect enhanced proliferation associated with decreased self-renewal and increased differentiation of LSCs in comparison to normal HSCs [14]. Unexpectedly, two members of MRC complex III, UQCRB and CTYB were downregulated. The reason for this effect is not known, but it may result from transcriptional regulation and/or better adaptation to bone marrow niche conditions [15-17]. Overall, elevated or normal expression of the components of MRC complex I and II suggests that OXPHOS reaction and electron flow can be properly initiated, but deregulated expression of the members of MRC complex III may "derail" and/or slow down the flow of electrons causing their "leakage". This 
speculation is supported by our previous report that Rac2-mediated reduction of $\Delta \Psi_{m}$ combined with inhibition of the electron flow through MRC complex III facilitated electron "leakage" resulting in high levels of ROS in CML-CP [5].

We reported before that imatinib inhibited BCR-ABL1 kinase and ROS in BCR-ABL1 positive 32Dcl3 cell line [12]. However, despite inhibition of BCR-ABL1 kinase, imatinib did not significantly reduce ROS in LSCs/LPCs in the presence of growth factors, suggesting that other factors play an important role in generation of high levels of ROS in patient cells [5]. Imatinib did not restore proper expression levels of MRC components in CML-CP LSCs implicating their continuous role in generation of ROS (this work) and accumulation of genomic instability even in imatinib-treated patients [3,13]. Retention of Rac2 activity in imatinib-treated CML-CP cells may contribute to this phenomenon. Functional studies are warranted to determine if postulated deregulation of the expression of MRC genes depends on Rac2 activity or other mechanisms.

Overall, our findings are consistent with the hypothesis that mitochondria present in CML-CP LSCs display a unique "metabolic-bioenergetic-oxidative" phenotype in which dysfunctional MRC generates high levels of mitochondrial ROS and low levels of ATP; the latter effect being compensated by enhanced glucose uptake and aerobic glycolysis [18]. CML-CP progression to CML-BP was accompanied with extensive downregulation of the components of MRC complex I and IV suggesting more pronounced defect. 


\section{Acknowledgements}

This work was supported in part by grants CA123014 and CA134458 to T.Skorski. D. Irvine is a Chief Scientist's Office Clinical Research Fellow; M. Copland is funded by the Scottish Funding Council (SCD/04) and Leukaemia and Lymphoma Research (No. 11017). Procurement of biospecimens was facilitated by the Glasgow Tissue Biorepository. This study was supported by the Glasgow Experimental Cancer Medicine Centre (ECMC), which is funded by Cancer Research UK and by the Chief Scientist's Office (Scotland).

\section{Declaration of Interests}

M. Copland has received research funding from Novartis and Bristol-Myers Squibb, is on Advisory Boards for Bristol Myers Squibb and Pfizer and has received honoraria from BristolMyers Squibb, Novartis and Pfizer. R. Bhatia has received honorarium from Novartis. The other authors declare no conflicts of interest. 


\section{References}

1. Melo JV, Barnes DJ. Chronic myeloid leukaemia as a model of disease evolution in human cancer. Nat Rev Cancer. 2007;7:441-53.

2. Corbin AS, Agarwal A, Loriaux M, Cortes J, Deininger MW, Druker BJ. Human chronic myeloid leukemia stem cells are insensitive to imatinib despite inhibition of BCR-ABL activity. J Clin Invest 2011;121:396-409.

3. Perrotti D, Jamieson C, Goldman J, Skorski T. Chronic myeloid leukemia: mechanisms of blastic transformation. J Clin Invest 2010;120:2254-64.

4. Slupphaug G, Kavli B, Krokan HE. The interacting pathways for prevention and repair of oxidative DNA damage. Mutat Res 2003;531:231-51.

5. Nieborowska-Skorska M, Kopinski PK, Ray R, et al. Rac2-MRC-clll-generated ROS cause genomic instability in chronic myeloid leukemia stem cells and primitive progenitors. Blood 2012;119:4253-63.

6. Zhang B, Strauss AC, Chu S, et al. Effective targeting of quiescent chronic myelogenous leukemia stem cells by histone deacetylase inhibitors in combination with imatinib mesylate. Cancer Cell 2010;17:427-42.

7. Subramanian A, Tamayo P, Mootha VK, et al. Gene set enrichment analysis: a knowledge-based approach for interpreting genome-wide expression profiles. Proc Natl Acad Sci U S A 2005;102:15545-50.

8. Diehn M, Cho RW, Lobo NA, et al. Association of reactive oxygen species levels and radioresistance in cancer stem cells. Nature 2009;458:780-3.

9. Ye XQ, Li Q, Wang GH, et al. Mitochondrial and energy metabolism-related properties as novel indicators of lung cancer stem cells. Int J Cancer 2011;129:820-31.

10. Abdel-Wahab O, Levine RL. Metabolism and the leukemic stem cell. J Exp Med 2010;207:677-80.

11. Koptyra M, Cramer K, Slupianek A, Richardson C, Skorski T. BCR/ABL promotes accumulation of chromosomal aberrations induced by oxidative and genotoxic stress. Leukemia. 2008;22:196972.

12. Koptyra $M$, Falinski $R$, Nowicki MO, et al. $B C R / A B L$ kinase induces self-mutagenesis via reactive oxygen species to encode imatinib resistance. Blood. 2006;108:319-27.

13. Skorski T. Chronic myeloid leukemia cells refractory/resistant to tyrosine kinase inhibitors are genetically unstable and may cause relapse and malignant progression to the terminal disease state. Leuk Lymphoma 2011;52 Suppl 1:23-9.

14. Petzer AL, Eaves CJ, Barnett MJ, Eaves AC. Selective expansion of primitive normal hematopoietic cells in cytokine-supplemented cultures of purified cells from patients with chronic myeloid leukemia. Blood. 1997;90:64-9.

15. Pihlajamaki J, Boes T, Kim EY, et al. Thyroid hormone-related regulation of gene expression in human fatty liver. J Clin Endocrinol Metab 2009;94:3521-9.

16. Xu F, Ackerley $\mathrm{C}$, Maj MC, et al. Disruption of a mitochondrial RNA-binding protein gene results in decreased cytochrome $\mathrm{b}$ expression and a marked reduction in ubiquinol-cytochrome $\mathrm{c}$ reductase activity in mouse heart mitochondria. Biochem J 2008;416:15-26.

17. Jung HJ, Shim JS, Lee J, et al. Terpestacin inhibits tumor angiogenesis by targeting UQCRB of mitochondrial complex III and suppressing hypoxia-induced reactive oxygen species production and cellular oxygen sensing. J Biol Chem 2010;285:11584-95.

18. Hitosugi T, Fan J, Chung TW, et al. Tyrosine phosphorylation of mitochondrial pyruvate dehydrogenase kinase 1 is important for cancer metabolism. Mol Cell 2011;44:864-77. 
Figure legends:

Figure 1. Expression of OXPHOS genes is altered in CML. (A) Differential expression (GO ANOVA) of genes involved in OXPHOS in stem and progenitor populations in CML and normal hematopoiesis; $\mathrm{n}$ = number of donors. (B) Heat map (One way ANOVA) identifying individual genes involved in OXPHOS which are significantly upregulated (red) or downregulated (blue) when compared to normal HSCs (gray). Results from HSCs and LSCs are highlighted in yellow boxes.

Figure 2. Expression of genes identified by the IFT "Oxidative Phosphorylation" is altered in CML. Heat map (One way ANOVA) identifying individual genes, which are significantly upregulated (red) or downregulated (blue) when compared to normal HSCs (gray). Results from HSCs and LSCs are highlighted in yellow boxes.

Figure 3. Expression of genes identified by the IFT "Mitochondrial Dysfunction" is altered in CML. Heat map (One way ANOVA) identifying individual genes, which are significantly upregulated (red) or downregulated (blue) when compared to normal HSCs (gray). Results from HSCs and LSCs are highlighted in yellow boxes.

Figure 4. Expression of genes encoding the components of MRC is altered in CML-CP LSCs and is not affected by imatinib. (A) Modified IPA of the expression of genes encoding MRC in CML-CP LSCs in comparison to normal HSCs. (B) Modified IPA of the expression of 
genes encoding MRC in the untreated versus imatinib-treated LSCs from CML-CP. Upregulated and downregulated genes are marked in red and blue, respectively. 

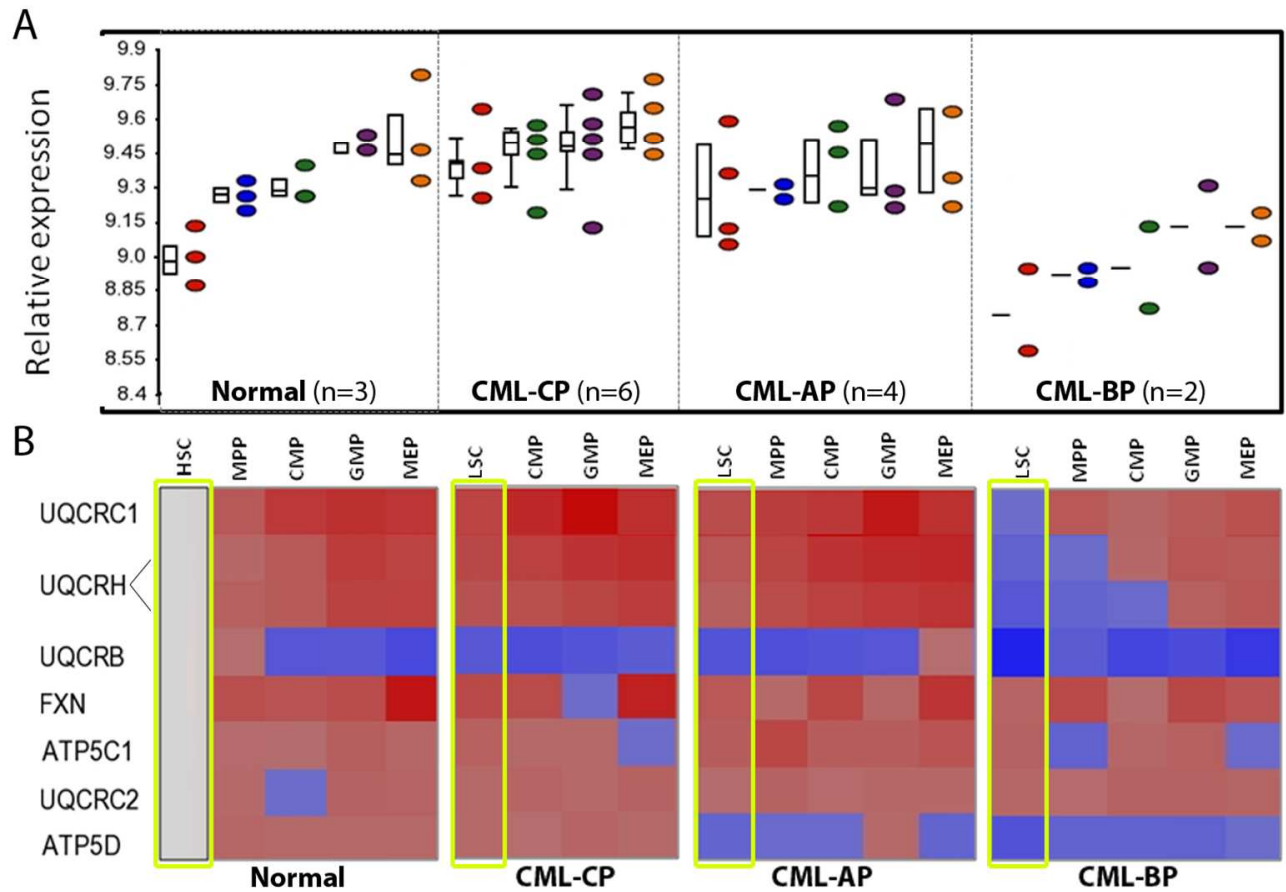

Figure 1 

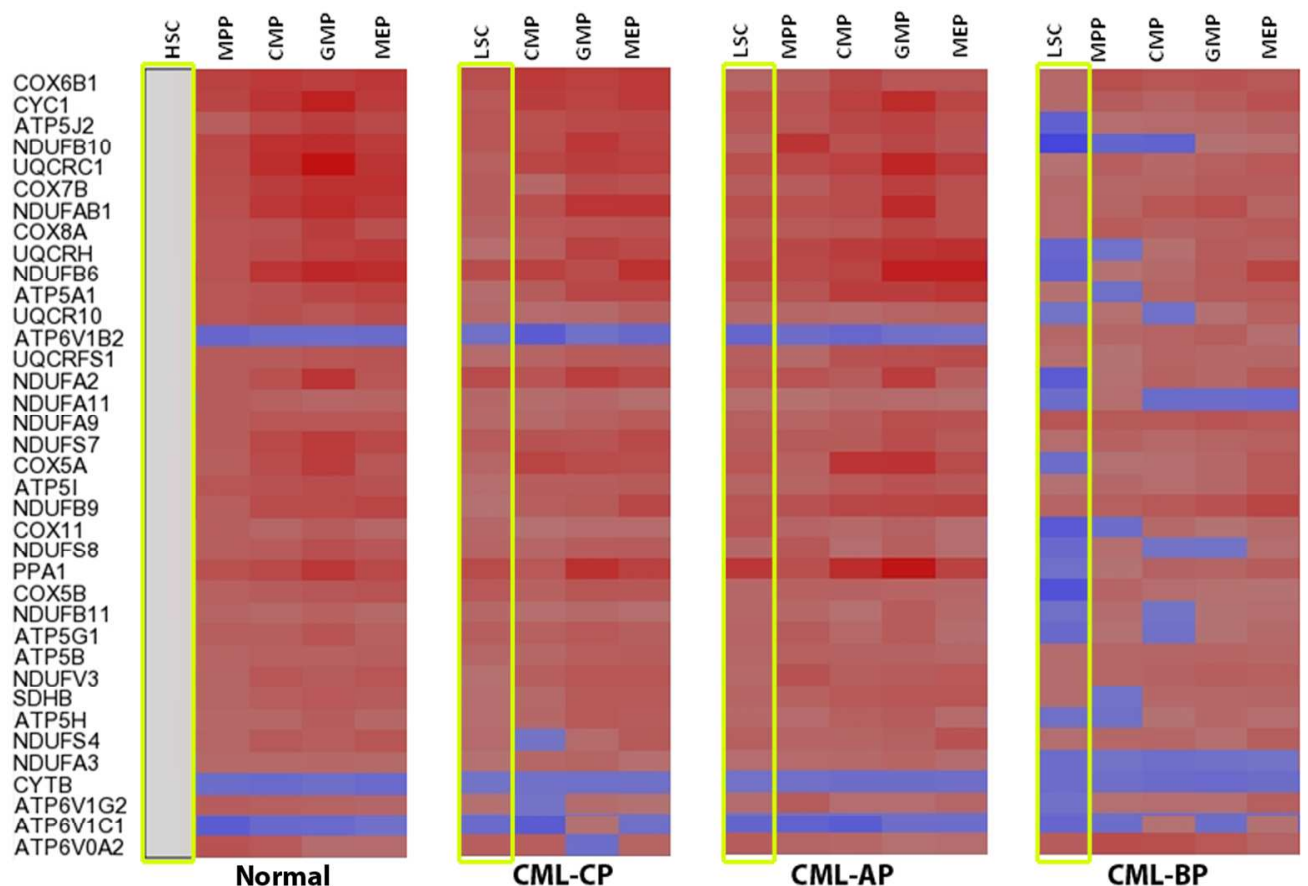

Figure 2 


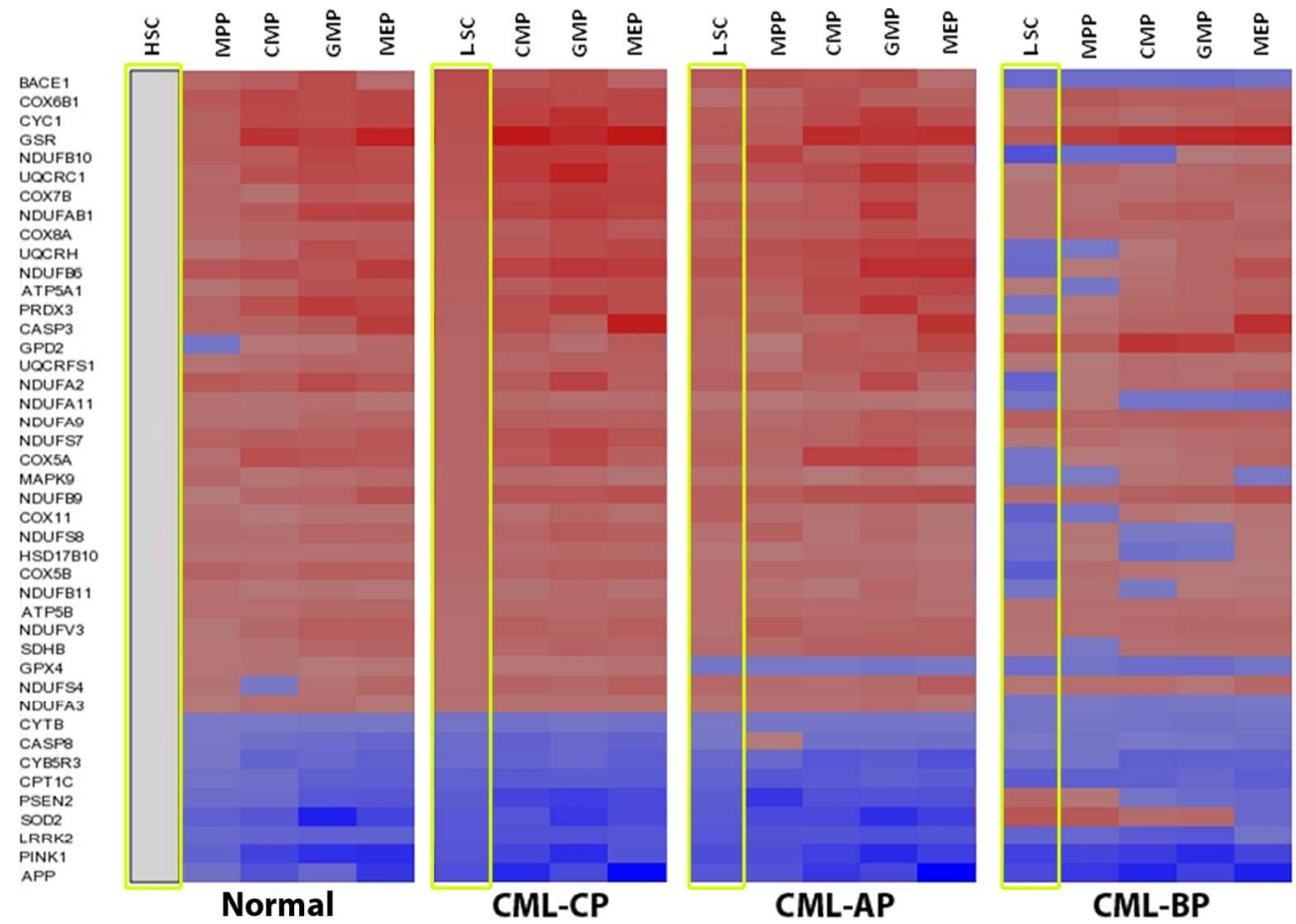

Figure 3 


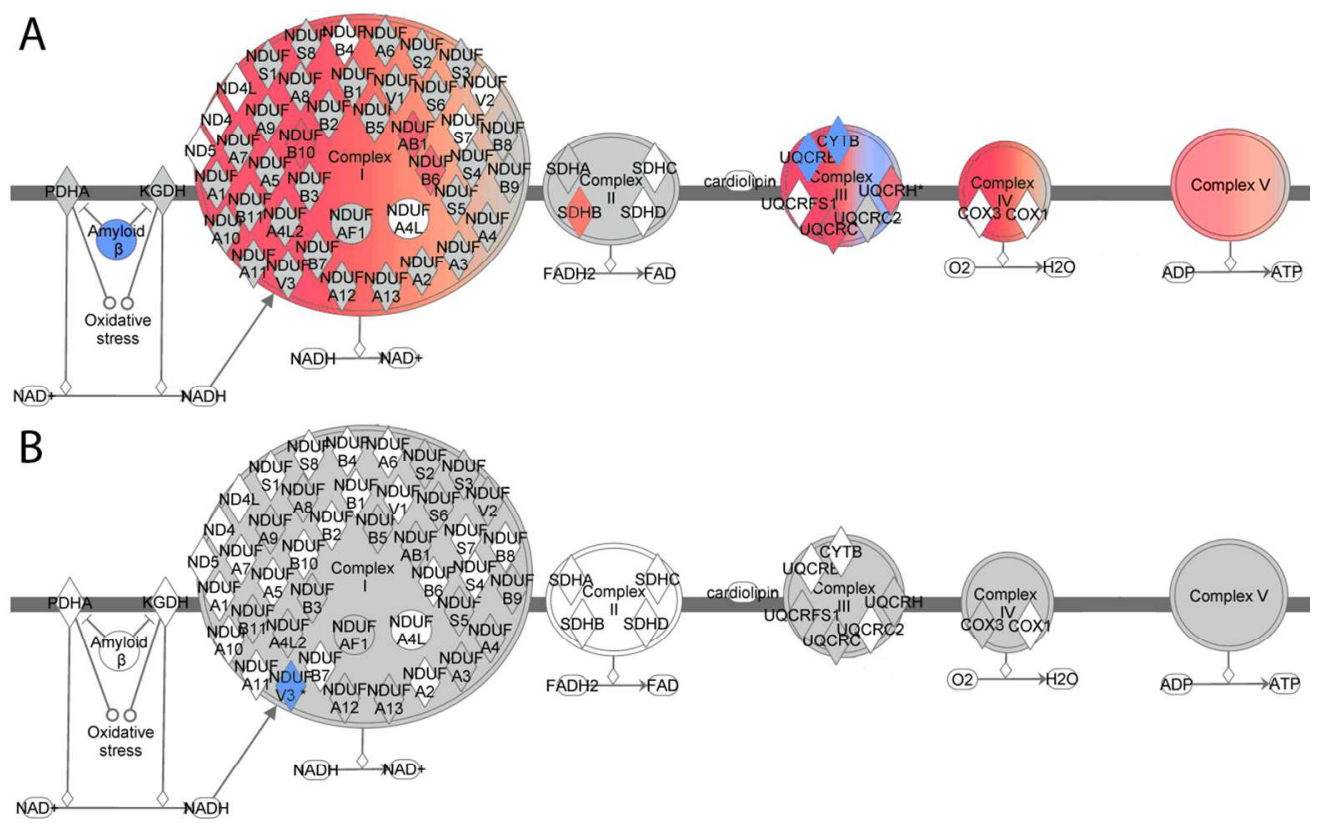

Figure 4 
Supplemental Table 1. Oxidative phosphorylation (GO-ID: 6119): CML-CP LSCs versus normal HSCs

\begin{tabular}{l|l|l} 
Gene & P value & Fold change \\
\hline UQCRC1 & 0.000654 & \\
\hline UQCRH & 0.006115 & 1.68953 \\
\hline FXN & 0.127043 & 1.60273 \\
\hline UQCRH & 0.015351 & 1.58157 \\
\hline ATP5C1 & 0.251449 & 1.44236 \\
\hline UQCRC2 & 0.425902 & 1.21661 \\
\hline ATP5D & 0.608306 & 1.08918 \\
\hline UQCRB & 0.039072 & 1.06473 \\
\hline
\end{tabular}


Supplemental Table 2. : Ingenuity Functional Term - "Oxidative phosphorylation" CMLCP LSCs versus normal HSCs

\begin{tabular}{|c|c|c|c|c|c|}
\hline Gene & $P$ value & Fold change & Gene & $P$ value & Fold change \\
\hline COX6B1 & $2.51 E-06$ & 1.87427 & ATP5I & 0.000165 & 1.51763 \\
\hline CYC1 & $3.36 \mathrm{E}-05$ & 1.82446 & NDUFB9 & 0.001193 & 1.37055 \\
\hline ATP5J2 & 0.007985 & 1.38781 & cox11 & 0.050168 & 1.36562 \\
\hline NDUFB10 & 0.015792 & 1.76151 & NDUFS8 & 0.044569 & 1.36554 \\
\hline UQCRC1 & 0.000136 & 1.75859 & PPA1 & 0.014709 & 1.60698 \\
\hline cox7B & 0.000308 & 1.69678 & COX5B & 0.094807 & 1.33068 \\
\hline NDUFAB1 & 0.003011 & 1.6692 & NDUFB11 & 0.007921 & 1.28617 \\
\hline COX8A & 0.000102 & 1.56086 & ATP5G1 & 0.005954 & 1.38345 \\
\hline UQCRH & 0.007048 & 1.55962 & ATP5B & 0.00081 & 1.27892 \\
\hline NDUFB6 & 0.011922 & 1.55013 & NDUFV3 & 0.01493 & 1.26489 \\
\hline ATP5A1 & 0.00226 & 1.50946 & SDHB & 0.014166 & 1.25953 \\
\hline UQCR10 & 0.000227 & 1.51849 & ATP5H & 0.043746 & 1.2302 \\
\hline ATP6V1B2 & 0.025858 & -1.23528 & NDUFS4 & 0.091942 & 1.24174 \\
\hline UQCRFS1 & 0.006097 & 1.43215 & NDUFA3 & 0.054158 & 1.19568 \\
\hline NDUFA2 & 0.013035 & 1.41154 & CYTB & 0.012989 & -1.12641 \\
\hline NDUFA11 & 0.020686 & 1.40854 & ATP6V1G2 & 0.002426 & 1.39659 \\
\hline NDUFA9 & 0.000335 & 1.40673 & ATP6V1C1 & 0.005832 & -1.39083 \\
\hline NDUFS7 & 0.01449 & 1.39788 & ATP6V0A2 & 0.005499 & 1.58971 \\
\hline COX5A & 0.070689 & 1.38152 & & & \\
\hline
\end{tabular}


Supplemental Table 3. : Ingenuity Functional Term "Mitochondrial Dysfunction" CML-CP LSCs versus normal HSCs

\begin{tabular}{|c|c|c|c|c|c|}
\hline Gene & P value & Fold change & Gene & $P$ value & Fold change \\
\hline BACE1 & 0.000289 & 1.88839 & NDUFB9 & 0.001193 & 1.37055 \\
\hline COX6B1 & 2.51E-06 & 1.87427 & Cox11 & 0.050168 & 1.36562 \\
\hline CYC1 & 3.36E-05 & 1.82446 & NDUFS8 & 0.044569 & 1.36554 \\
\hline GSR & $2.28 \mathrm{E}-05$ & 1.76463 & HSD17B10 & 0.005019 & 1.35441 \\
\hline NDUFB10 & 0.015792 & 1.76151 & COX5B & 0.094807 & 1.33068 \\
\hline UQCRC1 & 0.000136 & 1.75859 & NDUFB11 & 0.007921 & 1.28617 \\
\hline COX7B & 0.000308 & 1.69678 & ATP5B & 0.00081 & 1.27892 \\
\hline NDUFAB1 & 0.003011 & 1.6692 & NDUFV3 & 0.01493 & 1.26489 \\
\hline COX8A & 0.000102 & 1.56086 & SDHB & 0.014166 & 1.25953 \\
\hline UQCRH & 0.007048 & 1.55962 & GPX4 & 0.049485 & 1.248 \\
\hline NDUFB6 & 0.011922 & 1.55013 & NDUFS4 & 0.091942 & 1.24174 \\
\hline ATP5A1 & 0.00226 & 1.50946 & NDUFA3 & 0.054158 & 1.19568 \\
\hline PRDX3 & 0.002291 & 1.46893 & CYTB & 0.012989 & -1.12641 \\
\hline CASP3 & 0.025413 & 1.4658 & CASP8 & 0.036249 & -1.27424 \\
\hline GPD2 & 0.027559 & 1.44514 & CYB5R3 & 0.005975 & -1.28788 \\
\hline UQCRFS1 & 0.006097 & 1.43215 & СРT1C & 2.61E-05 & -1.4606 \\
\hline NDUFA2 & 0.013035 & 1.41154 & PSEN2 & 0.024165 & -1.57809 \\
\hline NDUFA11 & 0.020686 & 1.40854 & SOD2 & 0.053969 & -1.68882 \\
\hline NDUFA9 & 0.000335 & 1.40673 & LRRK2 & $1.48 \mathrm{E}-07$ & -1.70011 \\
\hline NDUFS7 & 0.01449 & 1.39788 & PINK1 & 0.000158 & -1.74063 \\
\hline COX5A & 0.070689 & 1.38152 & APP & 3.03E-06 & -1.85859 \\
\hline MAPK9 & 0.00297 & 1.37965 & & & \\
\hline
\end{tabular}


Supplemental Table 4.: MRC complex I-V gene expression levels in the untreated versus imatinib-treated CML-CP LSCs.

\begin{tabular}{|c|c|c|c|c|c|c|c|}
\hline Probeset ID & Gene & $P$ value & $\begin{array}{c}\text { Fold } \\
\text { change }\end{array}$ & Probeset ID & Gene & $P$ value & $\begin{array}{c}\text { Fold } \\
\text { change }\end{array}$ \\
\hline \multicolumn{8}{|c|}{ 1. Mitochondrial complex I: NADH dehydrogenase (ubiquinone) subunits } \\
\hline 202298_at & NDUFA1 & 0.867183 & 1.10158 & 203613_s_at & NDUFB6 & 0.466514 & 1.33051 \\
\hline 222971_at & NDUFA2 & 0.905607 & 1.01233 & 1559042_at & NDUFB6 & 0.277021 & -1.04994 \\
\hline 209223_at & NDUFA2 & 0.158368 & -1.30049 & 202839_s_at & NDUFB7 & 0.148330 & 1.12865 \\
\hline 209224_s_at & NDUFA2 & 0.937902 & -1.02965 & 211407_at & NDUFB7 & 0.486221 & -1.12377 \\
\hline 218563_at & NDUFA3 & 0.859866 & -1.09639 & 201227_s_at & NDUFB8 & 0.727341 & 1.23805 \\
\hline 217773_s_at & NDUFA4 & 0.819815 & 1.15072 & 214241_at & NDUFB8 & 0.739387 & -1.03214 \\
\hline 218484_at & NDUFA4L2 & 0.290043 & 1.11992 & 201226_at & NDUFB8 & 0.755049 & 1.12722 \\
\hline 215850_s_at & NDUFA5 & 0.477794 & 1.04157 & 222992_s_at & NDUFB9 & 0.821917 & 1.11209 \\
\hline 201304_at & NDUFA5 & 0.827338 & -1.15452 & 223112_s_at & NDUFB10 & 0.305749 & -1.15135 \\
\hline 202001_s_at & NDUFA6 & 0.672114 & 1.50434 & 228301_x_at & NDUFB10 & 0.714177 & -1.07149 \\
\hline 202000_at & NDUFA6 & 0.397883 & -1.31214 & 218320_s_at & NDUFB11 & 0.775565 & 1.05712 \\
\hline 1557532_at & NDUFA7 & 0.713307 & 1.06157 & 203478_at & NDUFC1 & 0.919001 & 1.06758 \\
\hline 202785_at & NDUFA7 & 0.665959 & -1.04726 & 232146_at & NDUFC1 & 0.760959 & -1.01626 \\
\hline 218160_at & NDUFA8 & 0.565271 & 1.33916 & 218101_s_at & NDUFC2 & 0.941795 & 1.05062 \\
\hline 208969_at & NDUFA9 & 0.850008 & -1.05655 & 222521_x_at & NDUFC2 & 0.855177 & -1.01445 \\
\hline 227206_at & NDUFA10 & 0.128318 & -1.08268 & 229647_at & NDUFS1 & 0.560758 & 1.13935 \\
\hline 1555548_at & NDUFA10 & 0.485857 & -1.12956 & 236356_at & NDUFS1 & 0.979558 & 1.00207 \\
\hline 1554719_at & NDUFA10 & 0.612086 & -1.05685 & 239268_at & NDUFS1 & 0.156351 & -1.03485 \\
\hline 217860_at & NDUFA10 & 0.727667 & -1.11972 & 235321_at & NDUFS1 & 0.682528 & -1.03471 \\
\hline 225304_s_at & NDUFA11 & 0.570616 & 1.07569 & 203039_s_at & NDUFS1 & 0.781332 & -1.12214 \\
\hline 228689_at & NDUFA11 & 0.546424 & -1.06260 & 201966_at & NDUFS2 & 0.356585 & 1.30053 \\
\hline 228690_s_at & NDUFA11 & 0.845222 & -1.12040 & 201740_at & NDUFS3 & 0.687337 & -1.24745 \\
\hline 223244_s_at & NDUFA12 & 0.772277 & 1.30788 & 209303_at & NDUFS4 & 0.512921 & 1.14782 \\
\hline 220864_s_at & NDUFA13 & 0.902751 & -1.05405 & 1555057_at & NDUFS4 & 0.281542 & -1.03651 \\
\hline 202077_at & NDUFAB1 & 0.773993 & 1.15994 & 201757_at & NDUFS5 & 0.769222 & 1.25458 \\
\hline 243630_at & NDUFB1 & 0.549958 & 1.04223 & 203606_at & NDUFS6 & 0.896025 & 1.11308 \\
\hline 206790_s_at & NDUFB1 & 0.611159 & 1.24158 & 242168_at & NDUFS7 & 0.500249 & 1.05153 \\
\hline 218200_s_at & NDUFB2 & 0.763360 & 1.20478 & 211752_s_at & NDUFS7 & 0.079138 & -1.09532 \\
\hline 240391_at & NDUFB2 & 0.196730 & -1.08277 & 232169_x_at & NDUFS8 & 0.900162 & 1.07497 \\
\hline 218201_at & NDUFB2 & 0.481261 & -1.06821 & 203190_at & NDUFS8 & 0.364762 & -1.24645 \\
\hline 203371_s_at & NDUFB3 & 0.506174 & -1.20467 & 203189_s_at & NDUFS8 & 0.703119 & -1.03706 \\
\hline 218226_s_at & NDUFB4 & 0.466680 & 1.64043 & 227795_at & NDUFV1 & 0.609766 & -1.12617 \\
\hline 228940_at & NDUFB4 & 0.901174 & 1.01031 & 208714_at & NDUFV1 & 0.782795 & -1.11417 \\
\hline \multirow[t]{2}{*}{ 203621_at } & NDUFB5 & 0.574450 & 1.25982 & 202941_at & NDUFV2 & 0.736427 & -1.26819 \\
\hline & & & & 226616_s_at & NDUFV3 & 0.021986 & -1.13033 \\
\hline
\end{tabular}

2. Mitochondrial complex II: succinate dehydrogenase subunits

\begin{tabular}{|l|l|r|r|}
\hline 201093_x_at & SDHA & 0.459680 & -1.27657 \\
\hline 202675_at & SDHB & 0.883282 & 1.03174 \\
\hline 214166_at & SDHB & 0.991117 & 1.00093 \\
\hline 202004_x_at & SDHC & 0.203899 & 1.40669 \\
\hline 210131_x_at & SDHC & 0.408984 & 1.12324
\end{tabular}

216591_s_at
215088_s_at
238056_at
215652_at
202026_at

SDHC

SDHC

SDHC

SDHD

SDHD

\begin{tabular}{|r|r|}
\hline 0.642960 & 1.04136 \\
\hline 0.945676 & 1.03904 \\
\hline 0.957705 & -1.00536 \\
\hline 0.908240 & 1.00738 \\
\hline 0.975438 & -1.00531 \\
\hline
\end{tabular}




\begin{tabular}{|c|c|c|c|c|c|c|c|}
\hline Probeset ID & Gene & $P$ value & $\begin{array}{l}\text { Fold } \\
\text { change }\end{array}$ & Probeset ID & Gene & P value & $\begin{array}{l}\text { Fold } \\
\text { change }\end{array}$ \\
\hline \multicolumn{8}{|c|}{ 3. Mitochondrial complex III: ubiquinol-cytochrome $\mathrm{c}$ reductase complex subunits } \\
\hline 201066 at & CYC1 & 0.587744 & -1.33051 & 201903 at & UQCRC1 & 0.607226 & -1.36365 \\
\hline 218190_s_at & UQCR10 & 0.964667 & 1.01081 & 212600_s_at & UQCRC2 & 0.848710 & 1.13128 \\
\hline 228142 at & UQCR10 & 0.898268 & -1.01408 & 241755 at & UQCRC2 & 0.203810 & -1.06954 \\
\hline 202090 s at & UQCR11 & 0.601351 & 1.56712 & 200883 at & UQCRC2 & 0.775845 & -1.26770 \\
\hline 223613_at & UQCR11 & 0.882728 & -1.02446 & 208909_at & UQCRFS1 & 0.639032 & -1.48297 \\
\hline 205849_s_at & UQCRB & 0.518622 & 1.64366 & 202233_s_at & UQCRH & 0.955300 & 1.03505 \\
\hline $209066 \times$ at & UQCRB & 0.542853 & 1.47041 & 201568 at & UQCRQ & 0.537262 & -1.38722 \\
\hline 209065_at & UQCRB & 0.985410 & 1.00445 & & & & \\
\hline 244293_at & UQCRB & 0.883126 & -1.00542 & & & & \\
\hline
\end{tabular}

4. Mitochondrial complex IV: cytochrome $c$ oxidase subunits

\begin{tabular}{|c|c|c|c|c|c|c|c|}
\hline 200086_s_at & COX4I1 & 0.400657 & 1.50342 & 201441_at & cox6B1 & 0.648072 & 1.23983 \\
\hline 202698_x_at & COX4I1 & 0.509855 & 1.64109 & 1553367_a_at & COX6B2 & 0.903653 & -1.02754 \\
\hline 213758_at & COX4I1 & 0.244363 & -1.16866 & 201754_at & cox6C & 0.806479 & -1.22889 \\
\hline 232761_at & COX412 & 0.674413 & -1.05780 & 204570_at & COX7A1 & 0.324356 & 1.11373 \\
\hline 203663_s_at & COX5A & 0.533099 & 1.67431 & 201597_at & COX7A2 & 0.963172 & -1.03472 \\
\hline 229426_at & COX5A & 0.896803 & 1.01955 & 201256_at & COX7A2L & 0.688165 & 1.20520 \\
\hline 211025_x_at & COX5B & 0.800752 & 1.06496 & 239252_at & CoX7B & 0.356746 & -1.29211 \\
\hline 202343_x_at & COX5B & 0.999996 & 1.00000 & 202110_at & COX7B & 0.787721 & -1.26278 \\
\hline 213736_at & COX5B & 0.135785 & -1.17547 & 231265_at & COX7B2 & 0.569338 & -1.07830 \\
\hline 213735_s_at & COX5B & 0.994309 & -1.00242 & 213846_at & coX7C & 0.519717 & -1.28219 \\
\hline 224344_at & COX6A1 & 0.372780 & 1.05732 & 217491_x_at & coX7C & 0.829521 & -1.21148 \\
\hline 200925_at & COX6A1 & 0.643732 & 1.23988 & 201134_x_at & coX7C & 0.946748 & -1.05321 \\
\hline 234876_at & COX6A1 & 0.358854 & -1.14119 & 201119_s_at & COX8A & 0.821015 & -1.07365 \\
\hline 206353_at & COX6A2 & 0.382106 & -1.16231 & 231487_at & CoX8C & 0.267065 & -1.04426 \\
\hline
\end{tabular}

5. Mitochondrial complex V: ATP synthase subunits

\begin{tabular}{|l|l|r|r|}
\hline 213738_s_at & ATP5A1 & 0.714095 & 1.34846 \\
\hline 1569891_at & ATP5A1 & 0.955786 & -1.00854 \\
\hline 201322_at & ATP5B & 0.746834 & -1.34473 \\
\hline 205711_x_at & ATP5C1 & 0.792557 & 1.27375 \\
\hline 213366_x_at & ATP5C1 & 0.827924 & 1.20394 \\
\hline 208870_x_at & ATP5C1 & 0.960874 & 1.04372 \\
\hline 214132_at & ATP5C1 & 0.568208 & -1.06196 \\
\hline 203926_x_at & ATP5D & 0.144014 & -1.18691 \\
\hline 213041_s_at & ATP5D & 0.315207 & -1.30603 \\
\hline 217801_at & ATP5E & 0.919716 & 1.09008 \\
\hline 230535_s_at & ATP5E & 0.388714 & -1.03942 \\
\hline 211755_s_at & ATP5F1 & 0.668729 & 1.70501 \\
\hline 243501_at & ATP5F1 & 0.681419 & 1.00573 \\
\hline 208972_s_at & ATP5G1 & 0.690204 & -1.24543 \\
\hline 208764_s_at & ATP5G2 & 0.838677 & 1.07683 \\
\hline 207552_at & ATP5G2 & 0.985030 & 1.00196 \\
\hline 207507_s_at & ATP5G3 & 0.761873 & 1.19014 \\
\hline 207508_at & ATP5G3 & 0.825819 & 1.24125 \\
\hline 228168_at & ATP5G3 & 0.899934 & -1.00806 \\
\hline
\end{tabular}

\begin{tabular}{|l|l|r|r|}
\hline 210149_s_at & ATP5H & 0.500637 & -1.28354 \\
\hline 1555998_at & ATP5H & 0.835896 & -1.03300 \\
\hline 209492_x_at & ATP5I & 0.805656 & 1.18158 \\
\hline 207335_x_at & ATP5I & 0.864985 & 1.08965 \\
\hline 202325_s_at & ATP5J & 0.962204 & -1.04835 \\
\hline 202961_s_at & ATP5J2 & 0.958668 & 1.05726 \\
\hline 1558179_at & ATP5J2 & 0.500469 & -1.06377 \\
\hline 208745_at & ATP5L & 0.112297 & 2.25655 \\
\hline 208746_x_at & ATP5L & 0.570229 & 1.52479 \\
\hline 210453 _at & ATP5L & 0.732132 & 1.29884 \\
\hline 207573_x_at & ATP5L & 0.751791 & 1.20839 \\
\hline 200818_at & ATP5O & 0.658107 & 1.56786 \\
\hline 216954_x_at & ATP5O & 0.877471 & 1.07834 \\
\hline 1564482_at & ATP5O & 0.255337 & -1.10591 \\
\hline 223338_s_at & ATPIF1 & 0.370273 & 1.32505 \\
\hline 218671_s_at & ATPIF1 & 0.750222 & 1.18601 \\
\hline 223339_at & ATPIF1 & 0.164763 & -1.18296
\end{tabular}

